\title{
Redução de Dimensionalidade para Reconhecimento de Padrões Eletromiográficos de Movimentos dos Dedos da Mão
}

\author{
Ana Cláudia Barbosa Rezende ${ }^{1}$, Henrique Pires Corrêa ${ }^{1}$, Flávio Henrique Teles Vieira ${ }^{1}$ \\ ${ }^{1}$ Escola de Engenharia Elétrica, Mecânica e de Computação \\ Universidade Federal de Goiás (UFG) \\ Av. Universitária, $\mathrm{n}^{o} .1488$ - Goiânia - GO - Brasil \\ cana@ufg.br, pires_correa@ufg.br, flavio_vieira@ufg.br
}

\begin{abstract}
The recognition of electromyographic signal patterns (EMG) is an important tool in the detection of abnormalities and prosthesis control. In addition, data projection and dimensionality reduction act directly on the classification, which can assist in the recognition of patterns. We propose the implementation and comparative analysis of the methods of linear discriminant analysis (LDA) and principal component analysis (PCA), together with the probabilistic classifier $K$ - nearest neighbor $(K N N)$, for the classification of a database of EMG signals of finger movements. Results point to a better performance of the $P C A$ together with the KNN in reducing the dimensionality and classification of EMG signals.
\end{abstract}

Resumo. O reconhecimento de padrões de sinais eletromiográficos (EMG) é ferramenta importante na detecção de anormalidades e controle de próteses. Ademais, a projeção dos dados e redução de dimensionalidade atuam diretamente na classificação, que pode auxiliar no reconhecimento de padrões. Propõe-se a implementação e análise comparativa dos métodos análise discriminante linear (LDA) e análise de componentes principais (PCA), em conjunto com o classificador probabilístico $K$ - vizinho mais próximo (KNN), para classificação de uma base de dados de sinais EMG de movimentos dos dedos da mão. Resultados apontam melhor desempenho do PCA em conjunto com o KNN na redução de dimensionalidade e classificação dos sinais EMG.

\section{Introdução}

Os sinais de eletromiografia de superfície (sEMG) têm sido utilizados em sistemas de controle mioelétrico e protético das mãos. Esses esforços concentram-se, principalmente, no que tange os movimentos dos dedos. Na verdade, para conseguir um dispositivo protético hábil, os movimentos dos dedos devem ser incluídos no sistema de controle (TENORE et al., 2008).

O reconhecimento de padrões é passo importante na caracterização correta dos sinais EMG. Muitos destes conjuntos de sinais possuem alta dimensionalidade, assim, torna-se desejável extrair de forma efetiva as informações compactadas nas múltiplas dimensões, e simultaneamente, favorecer a classificação e melhorar o sistema de reconhecimento de padrões (YAN et al., 2006). Alguns dos métodos de projeção e classificação 
de sinais incluem técnicas de aprendizado de máquina, como redes neurais artificiais, sistemas difusos (fuzzy) e modelagens probabilísticas (PCA ou Principal Component Analysis, LDA ou Linear Discriminant Analysis e classificador KNN ou KNearest Neighbors) (YOUSEFI; HAMILTON-WRIGHT, 2014)).

Khushaba et al. (2011) desenvolvem um método computacional melhorado de projeção de características de sinais sEMG para controle protético de movimentos combinados dos dedos da mão. O método baseia-se na análise de preservação de vizinhança difusa com decomposição QR (também conhecido como fatoração QR), denominado FNPA (Fuzzy Neighborhood Preserving Analysis), em sinais sEMG coletados por apenas dois eletrodos. Resultados práticos indicam a importância do FNPA em comparação a outros métodos de projeção, com uma precisão média de $91 \%$.

Os pesquisadores Zhang et al. (2014) apresentam um estudo comparativo sobre reconhecimento de padrão em sinais sEMG para uma mão robótica antropomórfica, com base nas técnicas de PCA e LDA. São implementados três esquemas diferentes de projeção (PCA, LDA e PCA + LDA) e o classificador de distância mínima (MDC ou Minimum Distance Classifier) é empregado na identificação de nove tipos de movimentos da mão. Os resultados indicam que a projeção PCA + LDA não apresenta melhora significativa em termos de desempenho no reconhecimento de padrões em comparação com a projeção realizada somente com o LDA.

Yang et al. (2019) propõem uma técnica que minimiza a sobreposição entre classes, que estão próximas umas das outras no espaço de entrada, no processo de redução de dimensão e projeção de características dos sinais. Com uma abordagem de redução de dimensionalidade supervisionada linear (SLDR ou Supervised Linear Dimensionality Reduction), a técnica denotada DR-MSP (Dimension Reduction via Maximizing Pairwise Separation Probability), realiza a maximização de separação da probabilidade dos pares para a redução de dimensionalidade em conjuntos de sinais de referência. Resultados práticos demonstram desempenho superior do DR- MSP em comparação a outros métodos SLDR para redução de dimensionalidade.

Devido à natureza de alta variação do sinal EMG, as informações depreendidas desses sinais tendem a se dispersar dentro do conjunto original (CHU; MOON; MUN, 2006). Os métodos de redução de dimensionalidade baseados na projeção das características demonstraram ser capazes de consolidar essas informações de modo efetivo (CHAN; GREEN, 2007). O uso desses também se justifica pela abordagem utilizada para extrair os sinais EMG de diferentes músculos. Geralmente os sinais são coletados por meio de múltiplos canais, levando a um grande conjunto de características que podem afetar a classificação e consequentemente dificultar o reconhecimento de padrões.

Esta pesquisa propõe um estudo comparativo de três métodos de redução de dimensionalidade de sinais sEMG de movimentos individuais e combinados dos dedos da mão. Especificamente, foram implementados os métodos PCA e LDA, para o processo de projeção das características dos sinais. Ademais, realizou-se a análise das taxas de erro de classificação e variância dos sinais em função da redução de dimensionalidade obtidas por cada uma das técnicas de projeção, PCA e LDA, em conjunto com o classificador probabilístico KNN. 


\section{Análise Discriminante Linear (LDA)}

O LDA é uma generalização do discriminante linear de Fisher, um método de reconhecimento de padrões e aprendizado de máquina para encontrar uma combinação linear de características que caracterizam ou separam duas ou mais classes de objetos ou eventos.

Dadas amostras de duas classes $C_{1}$ e $C_{2}$, para encontrar a direção, definida por um vetor $\omega$, de modo que quando os dados, $x$, são projetados em $\omega$, os exemplos das duas classes sejam separados o máximo possível. A projeção de $x$ na direção de $\omega$ é dada pela Equação 1 (ALPAYDIN, 2010):

$$
z=\omega^{T} x
$$

A Equação 2 define a matriz inter-classe $\left(S_{b}\right)$, enquanto as Equações 3 e 4 definem as matrizes intra-classe para cada uma das classes $\left(S_{1}\right.$ e $\left.S_{2}\right)$.

$$
\begin{gathered}
S_{b}=\left(m_{1}-m_{2}\right)\left(m_{1}-m_{2}\right)^{T} \\
S_{1}=\sum_{t=1}^{N} r^{t}\left(x^{t}-m_{1}\right)\left(x^{t}-m_{1}\right)^{T} \\
S_{2}=\sum_{t=1}^{N}\left(1-r^{t}\right)\left(x^{t}-m_{2}\right)\left(x^{t}-m_{2}\right)^{T}
\end{gathered}
$$

em que $x^{t}$ representa as amostras das classes, $m_{1}$ é a média das amostras da classe $C_{1}$, $m_{2}$ é a média das amostras da classe $C_{2}, N$ o número total de amostras, respectivamente. $r^{t}$ é um fator de classificação das amostras, em que $r^{t}=1$ caso $x^{t} \in C_{1}$ e $r^{t}=0$ caso $x^{t} \in C_{2}$. A matriz intra-classe total é calculada segundo a Eq. 5:

$$
S=S_{1}+S_{2}
$$

Assim, o vetor $\omega$ pode ser encontrado conforme apresenta a Eq. 6:

$$
\omega=c S^{-1}\left(m_{1}-m_{2}\right)
$$

em que $c$ é uma constante. Todavia, como o mais importante é a direção e não a magnitude de $\omega$, assume-se $c=1$.

Para o caso de $C$ classes, considerando $C>i>2$, as Equações 7 e 9 apresentam como calcular as matrizes inter-classe $\left(S_{b}\right)$, intra-classe $\left(S_{i}\right)$ da i-ésima classe:

$$
\begin{gathered}
S_{i}=\sum_{t} r_{i}^{t}\left(x^{t}-m_{i}\right)\left(x^{t}-m_{i}\right)^{T} \\
S=\sum_{i=1} S_{i}
\end{gathered}
$$




$$
S_{b}=\sum_{i=1} N_{i}\left(m_{i}-m\right)\left(m_{i}-m\right)^{T}
$$

em que $N_{i}=\sum_{t} r_{i}^{t}$. A matriz de dispersão inter-classes após a projeção é $W^{T} S_{b} W$ e a matriz de dispersão intra-classe após a projeção é $W^{T} S W$. Para maior dispersão inter-classes e menor dispersão intra-classes:

$$
J(W)=\frac{\left|W^{T} S_{b} W\right|}{\left|W^{T} S \quad W\right|}
$$

Os maiores autovetores de $S^{-1} S_{b}$ são a solução. $S_{b}$ é a soma de $K$ matrizes de ordem 1, a saber, $\left(m_{i}-m\right)\left(m_{i}-m\right)^{T}$, e apenas $C-1$ deles são independentes. Portanto, $S_{b}$ tem uma classificação máxima de $C-1$. Assim, define-se um novo subespaço, $(C-$ 1) - dimensional, onde o discriminante deve ser construído. Apesar do LDA utilizar a separabilidade de classes como seu critério de optimalidade, qualquer classificação ou método pode ser usado neste novo subespaço para estimar os discriminantes.

\section{Análise de Componentes Principais (PCA)}

A análise de componentes principais (PCA) é um método não supervisionado em que o critério a ser maximizado é a variância. O componente principal é $\omega_{1}$, de modo que as amostras, após a projeção para $\omega_{1}$, são espalhadas de forma que a diferença entre os dados se tornam mais aparentes. Para uma solução única e para tornar a direção o fator principal, tem-se $\left\|\omega_{1}\right\|=1$. Segundo a Equação 1 , se $z=\omega^{T} x$ e $\operatorname{Cov}(x)=$, então (ALPAYDIN, 2010):

$$
\operatorname{Var}\left(z_{1}\right)=\omega_{1}^{T} \quad \omega_{1}
$$

Busca-se $\omega_{1}$ de modo que $\operatorname{Var}\left(z_{1}\right)$ seja maximizado e restrito a $\omega_{1}^{T} \omega_{1}=1$. Escrevendo segundo um problema de Lagrange, tem-se a Equação 12:

$$
\max _{\omega_{1}} \omega_{1}^{T} \omega_{1}-\alpha\left(\omega_{1}^{T} \omega_{1}-1\right)
$$

A Equação 12 é válida se $\omega_{1}$ for um autovetor de e $\alpha$ o autovalor correspondente. Assim, seleciona-se o maior autovetor com o maior autovalor para a maximização da variância. Portanto, o componente principal é o autovetor da matriz de covariância dos dados de entrada com o maior autovalor, $\lambda_{1}=\alpha$.

O segundo componente principal, $\omega_{2}$, também deve maximizar a variância, ter comprimento unitário e ser ortogonal a $\omega_{1}$. Este último requisito é válido se após a projeção $z_{2}=\omega_{2}^{T} x$ não seja correlacionado com $z_{1}$. $\omega_{2}$ deve ser o autovetor de $\sigma$ com o segundo maior autovalor, $\lambda_{2}=\alpha$. Da mesma forma, pode-se demonstrar que as outras dimensões são dados pelos autovetores com autovalores decrescentes. O primeiro autovetor (aquele com o maior autovalor), $\omega_{1}$, ou seja, o componente principal, explica a maior parte da variância; o segundo explica o segundo maior; e assim sucessivamente. A Equação 1 pode ser redefinida como apresenta a Equação 13: 


$$
z=W^{T}(x-m)
$$

em que as $k$ colunas de $W$ são os $k$ autovetores principais do estimador para . Realizase a subtração da média das amostras $m$ de $x$ antes da projeção para centralizar os dados na origem. Após esta transformação linear, obtém-se um espaço $k$-dimensional cujas dimensões são os autovetores, os autovalores associados a estes autovetores representam as variâncias das amostras. Para normalizar as variâncias, realiza-se a divisão destas pelas raízes quadradas dos autovalores.

\section{Metodologia}

A base de dados utilizada contém medições de sinais EMG do antebraço de dez indivíduos adultos sem distúrbios neurológicos ou musculares (KHUSHABA et al., 2012).

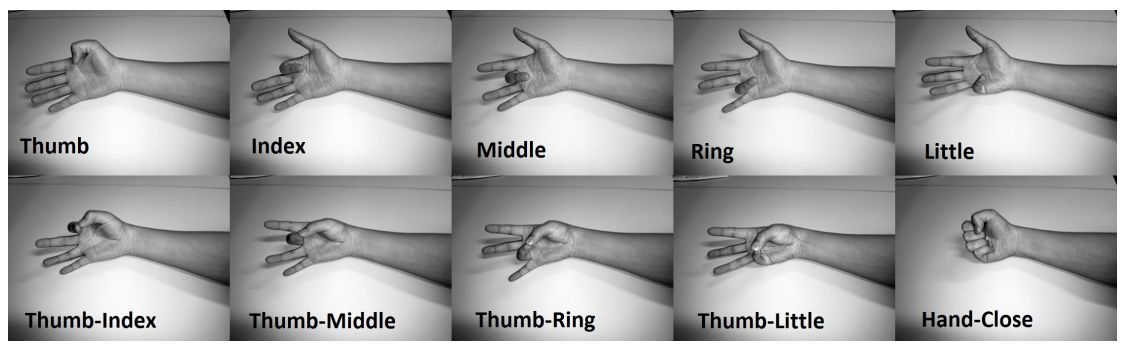

Figura 1. Dez classes de movimentos individuais e combinados dos dedos. Fonte: (KHUSHABA et al., 2012)

Dez movimentos individuais e combinados dos dedos foram realizados, como apresenta a Figura 1, incluindo a flexão de cada um dos dedos individuais, ou seja, polegar (Thumb), indicador (Index), médio (Middle), anelar (Ring), mínimo (Little) e o movimento de oposição entre os dedos polegar e indicador (Thumb-Index), polegar e médio (Thumb-Middle), polegar e anelar (Thumb-Ring), polegar e mínimo (Thumb-Little) e, finalmente, a mão fechada (Hand-Close). Foi solicitado que cada um dos dez indivíduos participantes realizassem seis repetições de cada um dos dez movimentos, estas repetições foram denominadas como observações.

A Figura 2 ilustra as etapas de processamento dos sinais sEMG até a obtenção dos dez movimentos classificados. Inicialmente todas as observações foram submetidas ao mesmo procedimento de segmentação, em uma etapa de janelamento do sinal com sobreposição.

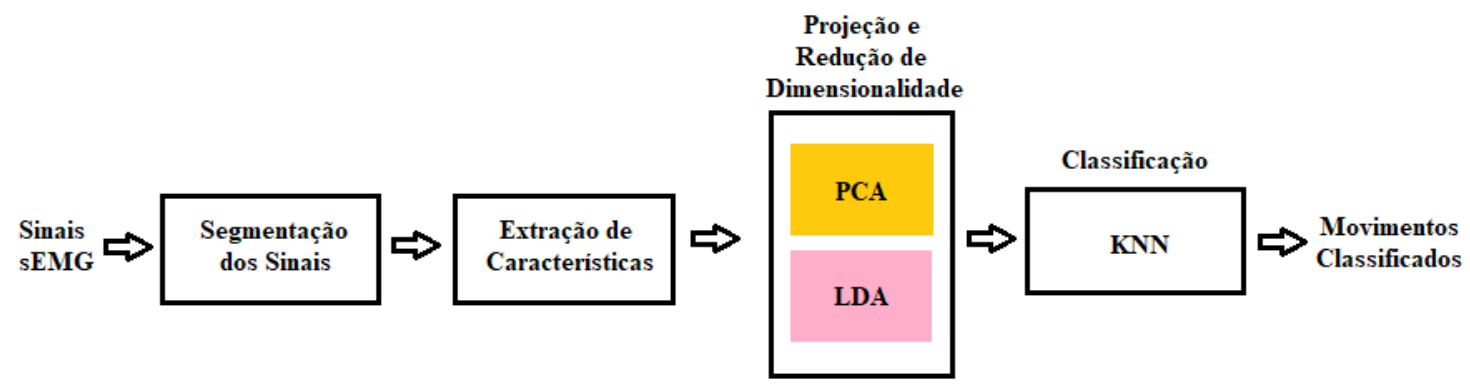

Figura 2. Diagrama das etapas de processamento dos sinais sEMG. 
Na próxima etapa, fase de extração de características, foram calculadas no domínio do tempo, valor médio absoluto (Mean Absolute Value ou MAV), alteração na inclinação do sinal (Slope Sign Change ou SSC), comprimento da forma de onda (Waveform Length ou WL) e número de cruzamentos em zero (Zero Crossing ou ZC) (HUDGINS; PARKER; SCOTT, 1993), raíz do valor quadrático médio (Root Mean Square ou RMS) (ENGLEHART; HUDGINS, 2003), integral de valor absoluto (Integral Absolute Value ou IAV) (SARIDIS; GOOTEE, 1982), descritores de Hjorth de atividade, mobilidade e complexidade (Hjorth Time Domain ou HTD) (HJORTH, 1970), assimetria (Skewness ou SS) (CACIOPPO; DORFMAN, 1987), e mais onze características provenientes do modelo Autoregressivo de ordem 11 (Autoregressive coefficients ou AR) (GRAUPE; CLINE, 1975), totalizando vinte e uma características. Posteriormente, todas essas características foram concatenadas em uma única matriz, definida como a matriz de entrada $X_{i n p}$, contendo todas as informações de todos os movimentos realizados por um mesmo indivíduo, ver Equação 14.

$$
\begin{array}{r}
X_{i n p}=\left[X_{1} X_{2} \ldots X_{21}\right] \\
Y_{i n p}=\left[Y_{1} Y_{2} \ldots Y_{10}\right]
\end{array}
$$

em que $X_{i}, i=1,2, \ldots, 21$, são vetores contendo os valores obtidos para cada uma das 21 características para todos os movimentos. Juntamente com $X_{i n p}$ foi definido um vetor de rótulos de mesmo comprimento, denominado $Y_{i n p}$, ver Equação 15. Cada um dos dez movimentos realizados durante a coleta foi denominado como uma classe, $Y_{1}$ rotula quais dados em $X_{\text {inp }}$ pertencem à classe $1, Y_{2}$ rotula os dados pertencentes à classe $2 \mathrm{e}$ assim sucessivamente. Esses vetores foram utilizados como entradas dos algoritmos de projeção e redução de dimensionalidade LDA e PCA. O cálculo dos vetores de projeção $\omega$ e dos vetores projetados $z$ foram realizados por meio das Equações referentes a cada um dos métodos e apresentados anteriormente nas Seções 2 e 3.

Os dados de entrada sofreram redução de dimensionalidade original, equivalente a 21 dimensões, para 20 dimensões, em seguida 19 dimensões, e assim sucessivamente até a redução a apenas 1 dimensão. Devido a restrição do LDA, de classificação máxima no subespaço $C-1$, ver Seção 2, o mesmo realizou redução de dimensionalidade de 21 para valores inferiores a 10 dimensões. A etapa de classificação dos sinais foi realizada pelo algoritmo $\mathrm{KNN}$, para quatro valores distintos de $\mathrm{K}$ vizinhos mais próximos, $\mathrm{K}=1$, $\mathrm{K}=3, \mathrm{~K}=5$ e $\mathrm{K}=7$.

Os índices utilizados para análise e comparação dos métodos de redução de dimensionalidade PCA e LDA foram as taxas médias de erro de classificação a variância entre as taxas de erro de classificação para cada indivíduo em função da dimensionalidade dos dados, utilizando o classificador KNN.

\section{Resultados}

A Tabela 1 apresenta um resumo das informações e parâmetros utilizados para a redução de dimensionalidade e classificação dos sinais sEMG da base de dados. A Figura 3 apresenta amostras de um dos sinais sEMG, no domínio do tempo, classificados nesta pesquisa. 
Tabela 1. Resumo das caracter sticas e parâmetros utilizados.

\begin{tabular}{c|c}
\hline \hline Tipo do sinal & EMG \\
Características do sinal & 21 \\
Tamanho da janela-sopreposição & $256-128 \mathrm{~ms}$ \\
Número de classes & 10 \\
Número de indivíduos & 10 \\
Algoritmos de Redução de Dimensionalidade & LDA e PCA \\
Algoritmo de Classificação & $\mathrm{KNN}(\mathrm{K}=1,3,5$ e 7) \\
\hline \hline
\end{tabular}
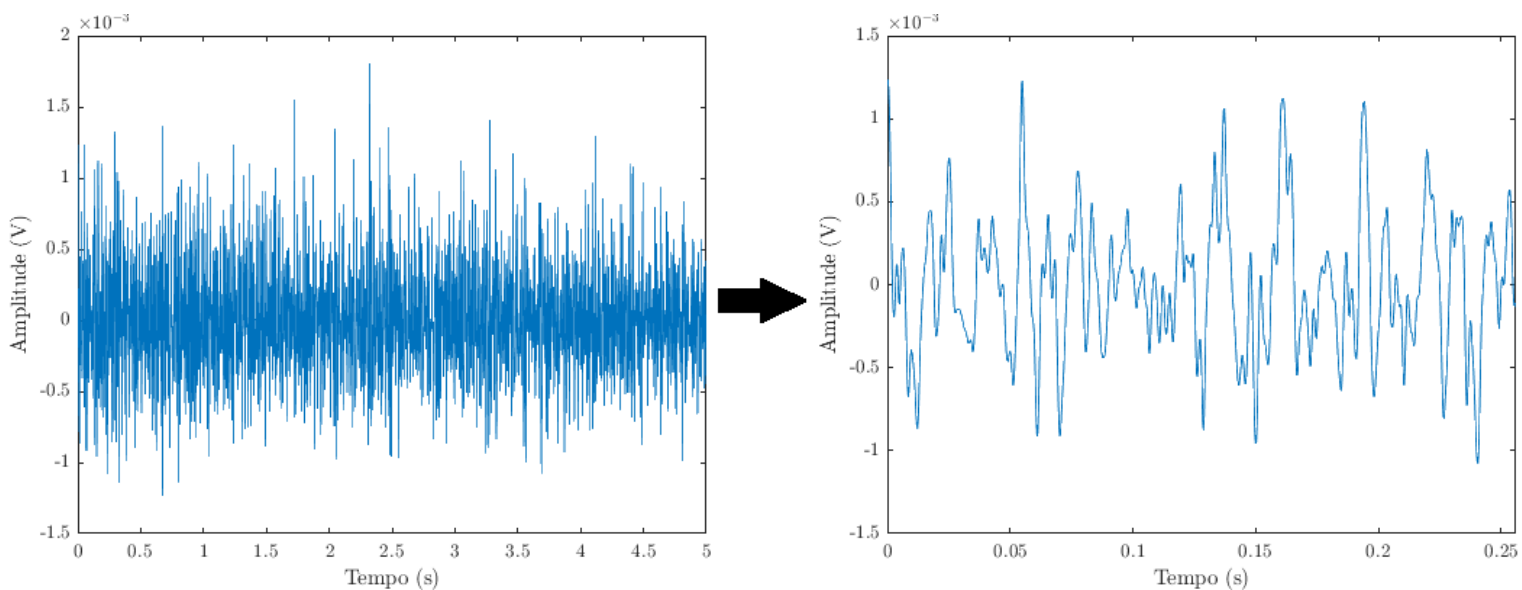

Figura 3. Amostras do sinal EMG no dom nio do tempo de um dos dez indiv duos da base de dados. A imagem esquerda apresenta o sinal sEMG inteiro coletado durante a realização de um dos dez movimentos dos dedos, citados previamente na Seção 4, em um intervalo de $5 \mathrm{~s}$. Na imagem direita é apresentada uma das segmentações do sinal, que foi janelado em intervalos de $256 \mathrm{~ms}$ com sobreposição de $128 \mathrm{~ms}$, para posteriormente passar pelo processo de extração de caracter sticas.

Reduzir os dados a apenas uma dimensão apresentou taxas médias elevadas de erro de classificação para todos os métodos avaliados, conforme apresentado nas Figuras 4 e 5. Isso demonstra a complexidade e importância das informações presentes nas múltiplas dimensões dos sinais, e também indica a dificuldade enfrentada no processo de redução de dimensionalidade em preservar as informações mais relevantes e significativas e projetá-las em um único subespaço ótimo.

O LDA apresentou pequena vantagem em relação ao PCA no processo de preservar informações relevantes dos sinais e reduzir dimensionalidade. Observou-se que para o intervalo entre duas a sete dimensões, o LDA apresentou menor taxa média de erro de classificação comparado ao PCA, utilizando os mesmos classificadores. Todavia, da perspectiva de melhor desempenho de classificação, o PCA em conjunto com o KNN alcançou menores taxas médias de erro de classificação para maiores dimensões, como pode-se visualizar entre o intervalo de dez a vinte dimensões. 


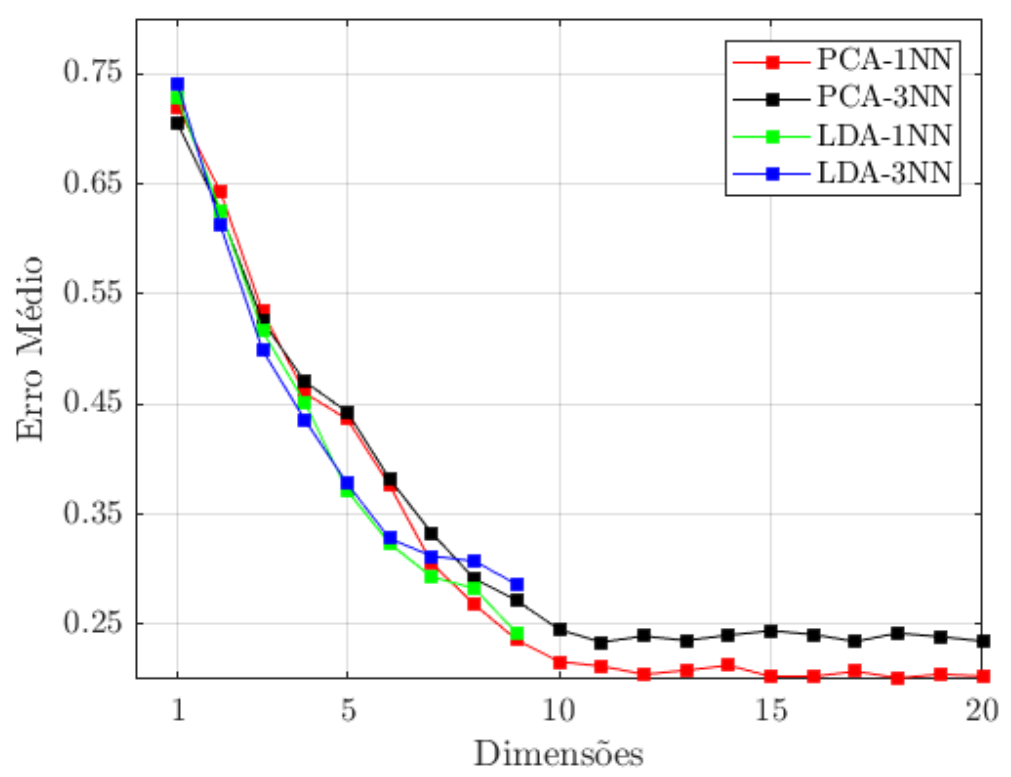

Figura 4. Taxa média de erro de classificação em função da dimensionalidade para PCA, LDA e KNN (K 1 e 3).

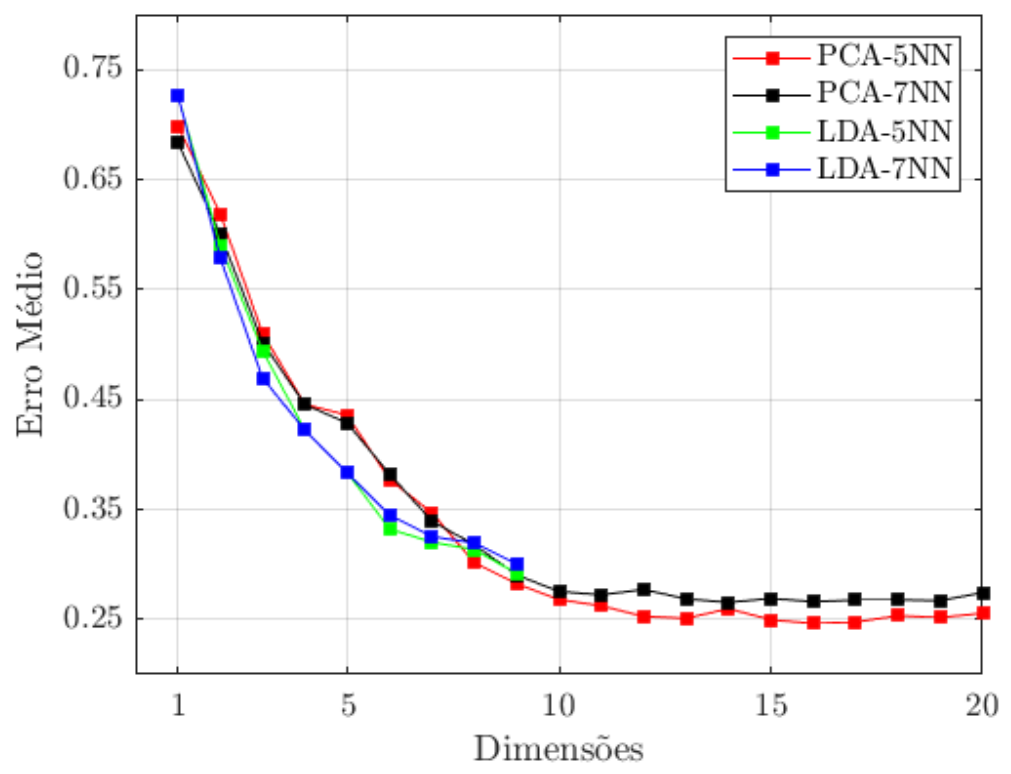

Figura 5. Taxa média de erro de classificação em função da dimensionalidade para PCA, LDA e KNN (K 5 e 7).

A Figura 6 apresenta a variância entre as taxas de erro de classificação para os dez indivíduos em função da dimensionalidade para os métodos PCA e LDA com o classificador $\mathrm{KNN}(\mathrm{K}=1,3,5$ e 7). O LDA foi o que apresentou maior variância, especificamente para o caso de nove dimensões. Entretanto para menor número de dimensões, intervalo de um a quatro, apresentou menor variância entre as taxas de erro de classificação obtidas para cada um dos dez indivíduos. O PCA apresentou queda na variância com o aumento de dimensionalidade. 

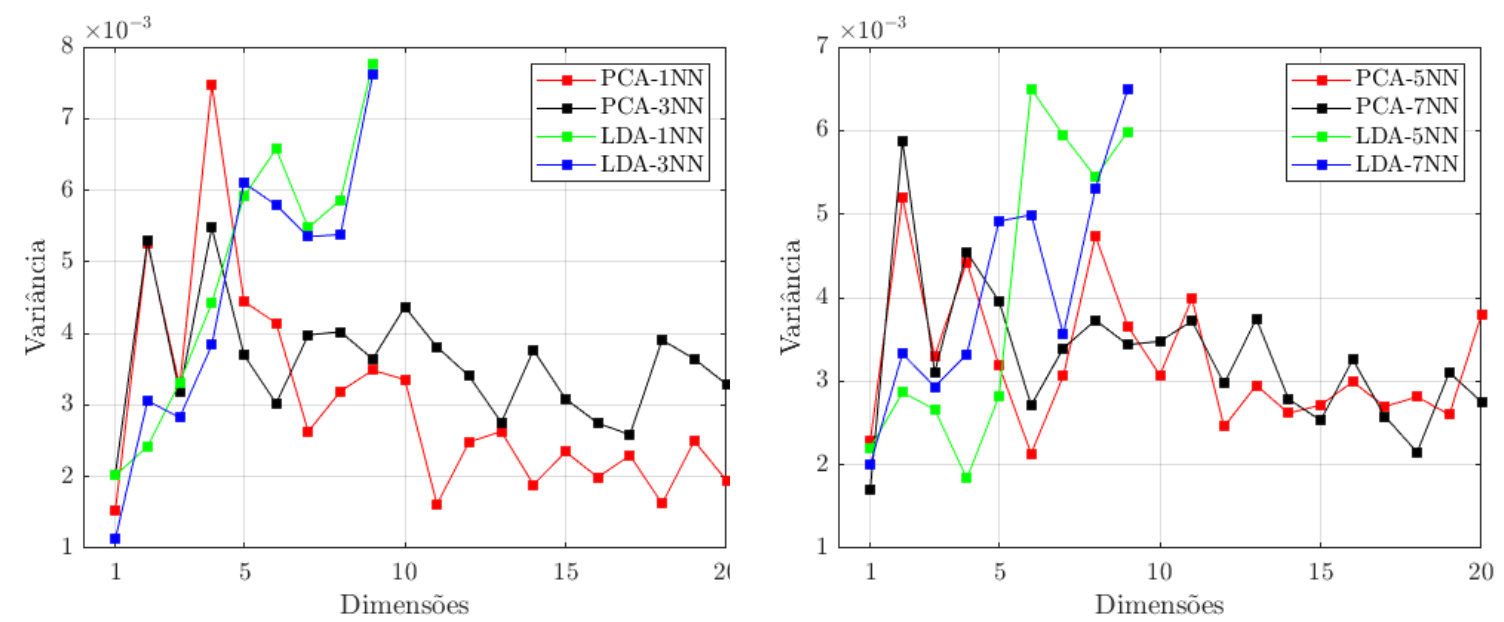

Figura 6. Variância entre as taxas de erro de classificação em função da dimensionalidade para PCA, LDA e KNN (K 1, 3, 5 e 7).

\section{Conclusões}

Dentre os métodos de redução de dimensionalidade avaliados, o PCA-KNN apresentou melhor desempenho comparado ao LDA-KNN, da perspectiva de ter alcançado as menores taxas médias de erro de classificação para maiores dimensões. O LDA também apresentou bom desempenho com relação à redução de dimensionalidade, inclusive superando o PCA em taxas médias de erro de classificação para menores dimensões.

A partir de aproximadamente 12 dimensões, as taxas médias de erro de classificação praticamente não se alteram, ou seja, o PCA pode reduzir de 21 para 12 dimensões, sem perdas relevantes de informações que possam prejudicar a classificação e ganhando em desempenho computacional. As menores taxas de erro de classificação foram obtidas para $\mathrm{K}=1$ no algoritmo de classificação KNN.

Como trabalhos futuros, pretende-se testar outros métodos supervisionados e nãosupervisionados de redução de dimensionalidade, como o FNPA e o DR-MSP. Ademais, explorar e comparar o desempenho de outras técnicas de classificação de sinais, como máquina de vetores de suporte (SVM ou Support Vector Machines) e a máquina de aprendizado extremo (ELM ou Extreme Learning Machine). Busca-se também ampliar e aprimorar a pesquisa dos sinais EMG de movimentos dos dedos da mão, incorporando e testando um número maior de bases de dados de sinais EMG da mesma natureza. De modo que ao final seja proposto um novo modelo computacional mais robusto e eficiente de projeção, redução de dimensionalidade e classificação de padrões de sinais EMG de dedos das mãos, para aplicações em próteses e sistemas de controle mioelétricos.

\section{Referências}

ALPAYDIN, E. Introduction to Machine Learning. [S.1.]: MIT Press, 2010.

CACIOPPO, J. T.; DORFMAN, D. D. Waveform moment analysis in psychophysiological research. Psychological Bulletin, American Psyc. Assoc., v. 102, n. 3, p. 421, 1987. 
CHAN, A. D.; GREEN, G. C. Myoelectric control development toolbox. CMBES Proceedings, v. 30, 2007.

CHU, J.-U.; MOON, I.; MUN, M.-S. A supervised feature projection for real-time multifunction myoelectric hand control. In: IEEE. 2006 International Conference of the IEEE Engineering in Medicine and Biology Society. [S.1.], 2006. p. 2417-2420.

ENGLEHART, K.; HUDGINS, B. A robust, real-time control scheme for multifunction myoelectric control. IEEE transactions on biomedical engineering, IEEE, v. 50, n. 7, p. 848-854, 2003.

GRAUPE, D.; CLINE, W. K. Functional separation of emg signals via arma identification methods for prosthesis control purposes. IEEE Transactions on Systems, Man, and Cybernetics, IEEE, n. 2, p. 252-259, 1975.

HJORTH, B. Eeg analysis based on time domain properties. Electroencephalography and clinical neurophysiology, Elsevier, v. 29, n. 3, p. 306-310, 1970.

HUDGINS, B.; PARKER, P.; SCOTT, R. N. A new strategy for multifunction myoelectric control. IEEE transactions on biomedical engineering, IEEE, v. 40, n. 1, p. 82-94, 1993.

KHUSHABA, R. N. et al. Electromyogram (emg) based fingers movement recognition using neighborhood preserving analysis with qr-decomposition. In: IEEE. 2011 Seventh International Conference on Intelligent Sensors, Sensor Networks and Information Processing. [S.1.], 2011. p. 1-105.

KHUSHABA, R. N. et al. Toward improved control of prosthetic fingers using surface electromyogram (emg) signals. Expert Systems with Applications, Elsevier, v. 39, n. 12, p. 10731-10738, 2012.

SARIDIS, G. N.; GOOTEE, T. P. Emg pattern analysis and classification for a prosthetic arm. IEEE Transactions on Biomedical Engineering, IEEE, n. 6, p. 403-412, 1982.

TENORE, F. V. et al. Decoding of individuated finger movements using surface electromyography. IEEE transactions on biomedical engineering, IEEE, v. 56, n. 5, p. 1427-1434, 2008.

YAN, S. et al. Graph embedding and extensions: A general framework for dimensionality reduction. IEEE transactions on pattern analysis and machine intelligence, IEEE, v. 29, n. 1, p. 40-51, 2006.

YANG, L. et al. Nonparametric dimension reduction via maximizing pairwise separation probability. IEEE Transactions on Neural Networks and Learning Systems, IEEE, v. 30, n. 10, p. 3205-3210, 2019.

YOUSEFI, J.; HAMILTON-WRIGHT, A. Characterizing emg data using machinelearning tools. Computers in biology and medicine, Elsevier, v. 51, p. 1-13, 2014.

ZHANG, D. et al. A comparative study on pca and lda based emg pattern recognition for anthropomorphic robotic hand. In: IEEE. 2014 IEEE International Conference on Robotics and Automation (ICRA). [S.1.], 2014. p. 4850-4855. 